\title{
La evolución de la seguridad en el sistema internacional contemporáneo: un balance a partir de los casos de Estados Unidos y la Federación de Rusia
}

\author{
Sara Patricia Quintero Cordero \\ Miguel Antonio GonZález Martínez
}

\section{Introducción}

El fin de la Guerra Fría marcó una nueva ruptura con los patrones que habían caracterizado la mayor parte del periodo posterior a la Segunda Guerra Mundial, en lo que respecta a las relaciones internacionales. Los cambios y las turbulencias en el panorama internacional, producto del vacío de poder, anunciaban que la transición hacia un nuevo orden mundial relativamente estable, como el anterior, requeriría tiempo para que los polos de poder se reacomodaran, en la medida en que las imágenes que se proyectaban hacia el ordenamiento de la sociedad internacional diferían con respecto al papel que desempeñaba Estados Unidos como cabeza de este nuevo orden.

Otro elemento importante yuxtapuesto, que suscita aún controversia en la categorización de la transición del sistema internacional a su versión "contemporánea" es la preponderancia del Estado-nación como el actor protagonista de las relaciones internaciones (Ohmae, 1997). A falta de una hegemonía continua de las grandes potencias, el surgimiento de Estados regionales — siguiendo la línea del Ohmae- y 
los nuevos actores de las relaciones internacionales: las organizaciones no gubernamentales e intergubernamentales, parecen adaptarse con más celeridad a la lógica del nuevo sistema (Pearson y Rochester, 2000).

Esta coyuntura de caos del sistema internacional en transición sirvió de escenario para el surgimiento de un nuevo enfoque de la seguridad. Aunque la propuesta emergió en el mundo académico en la década de los años ochenta, cobró importancia con el auge de las ideas liberales (Peréz de Armiño, 2006). Así, la doctrina clásica de seguridad nacional ${ }^{1}$ ha ido dejando paso a la seguridad humana, una expresión que considera que el individuo debe ser objeto de protección ante amenaza cada vez más globales y complejas (Bassedas, 2012).

En aras de contribuir a los estudios de paz y seguridad, el presente capítulo propone una revisión a la incorporación y la evolución de las nuevas amenazas emergentes de acuerdo con el concepto clásico de la seguridad en las principales potencias mundiales, es decir, se pretende establecer que la seguridad internacional en ese sistema contemporáneo sigue teniendo grandes visos clásicos del concepto. Se analizarán los casos de Estados Unidos y Rusia, como los grandes poderes del sistema internacional contemporáneo, para establecer cómo se ha adaptado el nuevo enfoque a la política de seguridad en cada país incorporando nuevos elementos, como el de la seguridad humana, sin que evolucione totalmente este concepto en las relaciones internacionales.

Este enfoque estatocéntrico descansa en la premisa teórica de que a pesar que los nuevos paradigmas de la seguridad fueron esbozados a partir de la idea de la cooperación y la interdependencia entre los diversos actores del sistema internacional, la observación empírica compagina con los planteamientos neorrealistas de las Relaciones Internacionales en los que aún los Estados conservan el papel protagonista en la dirección de la agenda de la seguridad internacional.

1 Desde la Segunda Guerra Mundial, Estados Unidos adoptó el concepto de seguridad nacional como un elemento clave en su política exterior. Esta prioridad aumento con la transición de la posguerra, cuando la Guerra Fría sentó las bases de un orden internacional bipolar, periodo en el cual se consolidó la defensa de la soberanía y la integridad territorial del Estado. 


\section{Hacia un nuevo enfoque de la seguridad}

El cambio del enfoque de la seguridad nacional hacia el individuo proyecta la idea del abandono del monopolio de los Estados sobre la seguridad internacional. Sin embargo, tanto el uso como la aceptación del nuevo enfoque de la seguridad humana aún está pendiente en el ejercicio de las relaciones internacionales. El nuevo enfoque tiene raíces en la década de los años ochenta, en diferentes círculos académicos; el aumento y la diversificación de la naturaleza de los actores en la escena internacional alimentó la necesidad de replantear el modelo estatocéntrista de la seguridad (modelo clásico), en el cual, la supervivencia y la soberanía de las principales unidades del sistema internacional debía prevalecer. El fin de la Guerra Fría, que supuso la disminución de los enfrentamientos en el mundo derivado de las tensiones políticas e ideológicas ante el auge de las ideas liberales, sirvió de plataforma de lanzamiento del nuevo enfoque de seguridad. Así para Pérez de Armiño (2006) el cuestionamiento del paradigma tradicional ha girado sobre todo en torno a dos ejes:

El primero ha consistido en el cuestionamiento del contenido de la seguridad y en el ensanchamiento del mismo, incorporando a las amenazas militares otras de tipo económico, político, social, medioambiental, etc. Este proceso ha conllevado un acercamiento entre las cuestiones de seguridad y las de desarrollo, que se ha incrementado en los años noventa y se ha reflejado en el concepto de seguridad humana. El segundo eje de evolución ha girado en torno al objeto de la seguridad, pasándose de la visión estatocéntrica clásica a las visiones que se centran en un nivel global o individual. Esta última perspectiva nos interesa particularmente, pues es la que acaba desembocando en la aparición del concepto de seguridad humana. (Pérez de Armiño, 2006, p 76)

Otro elemento determinante que potenció el nuevo enfoque de la seguridad fue el advenimiento de la revolución tecnológica en los años noventa y la interconexión que esta situación creó (Ariza, 2010). La idea del mundo globalizado, afianzó la interdependencia que los Estados aumentaron con la difuminación de las fronteras físicas. Así, la interrelación 
comercial, el daño sistemático al medioambiente y las enfermedades epidemiológicas, entre otros temas, se constituyeron como las fuentes de las nuevas amenazas potenciales a la seguridad mundial.

La propuesta se materializó con el aporte del paradigma del desarrollo humano, según el cual se debe garantizar el ensanchamiento de las capacidades y las libertades de las personas, en contraposición al concepto clásico del desarrollo, entendido en términos de mero crecimiento macroeconómico (Peréz de Armiño, 2006). El Informe sobre Desarrollo Humano de 1994 del Programa de las Naciones Unidas para el Desarrollo (PNUD, 1994) —más adelante, apoyado por la Comisión de la Seguridad Humana, liderada por Japón, publicado en 2003- afirma que es preciso abordar la lucha por la paz desde dos frentes interconectados: el primero hace referencia a la libertad ante los miedos, es decir, la eliminación del uso de la fuerza y de la violencia — así como la amenaza de su uso- de la vida diaria de las personas, y el segundo, a la libertad ante las necesidades: o, dicho de otra manera, el establecimiento de las condiciones de vida necesarias en términos económicos, alimenticios, sociales, ambientales y de salud (Bassedas, 2012).

No obstante, poco tiempo después, las dinámicas de transición del sistema visibilizaron las dificultades para implementar efectivamente el nuevo enfoque de seguridad. Si bien la publicación del Informe de Desarrollo Humano estableció la creciente interdependencia y la complejidad de las amenazas a seguridad, los atentados terroristas del 9-11 en Estados Unidos abrieron nuevamente el debate hacia el retorno del Estado como el garante de la seguridad internacional. Un año después de los ataques en Nueva York y Washington D.C. el presidente de Estados Unidos, George W. Bush (2002-2010), publicó una estrategia de seguridad nacional, la cual, para Bassedas (2012), en buena parte, entra en contradicción con los principios básicos de la doctrina de seguridad humana. La National Security Strategy (2002), al mismo tiempo que estableció los ataques militares preventivos como mecanismo de respuesta a las amenazas, también priorizó las acciones unilaterales, al considerar que no es imprescindible una autorización previa del Consejo de Seguridad de las Nacionales Unidas para iniciar una operación militar. 
Esta posición se traduce en el retorno hacia la visión clásica de la seguridad en el sistema internacional, con una variable adicional propia de los años de la globalización: la trasformación de la amenaza. El problema ya no son los ejércitos enemigos que quieren invadir y ocupar el territorio estadounidense (Estados Unidos, 2002), sino que los actores que serán objetivo de la política exterior de Estados Unidos son los terroristas, los Estados donde actúan y los rogue states, o Estados canallas, y el argumento es que estos Estados apoyan el terrorismo internacional (Bejer, 2005). De este modo, las acciones militares de Estados Unidos en Afganistán (2001) y en Irak (2003) han marcado el retorno hacia acciones que parecen más ajustadas a la visión del Estado, como el eje central de la seguridad internacional, llevándolo al uso de los medios tradicionales para defender su interés nacional. ${ }^{2}$ La globalización ha llevado a que los ajustes de las agendas de política de exterior de las potencias se alienen a la respuesta al terrorismo trasnacional, en menoscabo a las repuestas de las amenazas derivadas del enfoque de seguridad humana.

\section{Acercamiento teórico}

Una interpretación teórica ajustada al papel que desempeña el Estado y sus políticas de seguridad en el mundo interdependiente se pueden encontrar en los análisis de Kenneth Waltz (2008), quien propone que el marco estratégico de mejoramiento de la seguridad nacional del Estado se apoya en el principio de autoayuda. Así, en condiciones de anarquía — sin un poder central en el sistema internacional- la seguridad del Estado depende exclusivamente de las acciones que los Estados puedan emprender (Sodupe, 2008). Si bien, desde este enfoque el estallido de guerras se convierte en una amenaza latente, los Estados comienzan a afianzar su seguridad con políticas que tienen un gran componente militar; en otras palabras, dentro de la estrategia de defensa y seguridad de un Estado el componente tecnológico militar, y sus factores, refuerzan la seguridad misma no solo del Estado, sino

2 Esta particularidad será analizada más en detalle en caso de Estados Unidos. 
también de los propios ciudadanos y afianza la continuidad, y el statu quo del sistema. Los grandes polos de poder se dejan influenciar en sus políticas de defensa, en el actuar, el diseño y la innovación tecnológica de otras potencias, dando paso a retracciones positivas como lo afirmara Reynolds (1978) que un actor "A" incide en la política de seguridad del actor "B", dependiendo del actuar siempre del primer actor.

En este sentido, una conclusión preliminar es que la seguridad se origina propiamente del Estado. A pesar de que las nuevas amenazas, o como lo afirma Hoffman, las "nuevas guerras" en las que el monopolio de la guerra se pierde con otros actores del sistema, la seguridad sigue siendo demanda de esa estructura política y social llamada Estado, no obstante, la globalización y que esta origine nuevas entidades mundiales como las "comunidades de seguridad", que parte del supuesto que la interdependencia ensancha el concepto de seguridad nacional.

Pareciese entonces que, al denominar seguridad no solo en términos nacionales, su interés de dependencia mutua por combatir amenazas comunes reivindica la seguridad nacional, y se torna así un tema nacional. El soporte empírico está mercado por la evolución de las fuerzas militares en el ámbito internacional, que continúan preparando doctrinas insertando las nuevas amenazas y enfrentándolas desde una doctrina fundamentada en lo militar, combinando otros factores no militares visto desde lo meramente clásico. Por ende, el tema de la seguridad se toma desde un análisis de la estrategia militar, donde aspectos: defensa, disuasión, carrera y control de armamentos y desarme, y otros conceptos como alianzas militares, poder, paz, terrorismo, se articulan desde el Estado con el discurso de un nuevo enfoque de seguridad o seguridad humana, que no deja de ser clásico para revertirlo a un nuevo enfoque.

De esta manera se podrá observar cómo los grandes polos de poder actual (Estados Unidos, Rusia y China) reconocen amenazas dentro de sus políticas, por lo cual pretenden adoptar políticas comunes globales sin dejar de perseguir los intereses particulares de cada uno. El nuevo concepto de seguridad humana que emerge se consolidará si existe también un fortalecimiento de la seguridad nacional y de la supervivencia del Estado dentro del sistema internacional. Al respecto, Buzan (1992) intenta encontrar nuevos argumentos de la seguridad 
global al afirmar que las amenazas comunes globales podrían ser combatidas de una manera cooperativa, pero soportado desde el Estado, no desconociendo otros actores del sistema y que la realidad de la seguridad internacional no puede ser soportada en el Estado. Buzan sustenta su análisis de seguridad en el concepto denominado complejos de seguridad, en el cual un Estados, al tener una amenaza igualmente común con otros Estados, formará un complejo para reducirla o exterminarla, siempre y cuando no exista un poder dominante dentro de dicho complejo, puesto que, como afirma Waltz (1988), las grandes potencias siempre intentarán no depender de otros actores del sistema o controlar aquello por lo que dependen, queriendo siempre ampliar su grado de control y su autosuficiencia (Sodupe 2008).

\section{El periodo. El sistema internacional contemporáneo}

Difícilmente se puede hallar una conceptualización específica sobre la periodización del sistema internacional y sus implicaciones para los análisis. Aun cuando Francis Fukuyama proclamó tempranamente, el "fin de la historia", respaldado en la democratización del mundo con el triunfo de las ideas liberales (Fukuyama, 1995), otros autores, como Wallerstein, argumentan que el vacío de poder en el sistema actual aún persiste, al punto de hablar de un "caos" o de un "des-orden" mundial rampante. ${ }^{3}$ Siguiendo a Wallerstein, él sostiene que si bien a destrucción del muro de Berlín en 1989 y el desmembramiento de la Unión Soviética representó la caída del comunismo y el derrumbe del marxismo-leninismo, estos hechos no se pueden interpretar como el triunfo definitivo del liberalismo (Wallerstein, 2001).

El presente estudio proyecta un análisis de los principales actores (potencias) del sistema internacional contemporáneo. Partimos de la premisa que el des-orden que impera en el sistema internacional contemporáneo es derivado del vacío de un poder hegemónico en el sistema,

3 Para ver más sobre la idea de caos, antiorden o des-orden en el sistema internacional, ver en (Ghotme, 2010). 
aún sigue siendo dominado - y en algunos casos controlado— por los actores más dominantes: Estados Unidos, Rusia y China, estos últimos como poderes emergentes (Ghotme, 2010). Esta posición dual —de líderes y de antagonistas en el sistema- ha impedido el acomodamiento de China y Rusia al interés de Estados Unidos de ejercer una posición dominante en el sistema; ${ }^{4}$ y precisamente, un reflejo de esta posición es la falta de adhesión de las potencias emergentes al enfoque de seguridad basada en el individuo. Por lo tanto, en este plano de transición del sistema, las actuaciones de los Estados hoy aparecen ajustadas al plano de comprensión de los enfoques clásicos, donde la lucha por el poder sigue siendo un factor vigente en la proyección de las amenazas.

Si bien, el Sistema Contemporáneo advierte en una periodización compleja, hay que recalcar, justamente, que sus características fueron las que han dado luz para ensanchar el espectro de la seguridad, en la medida en que se lograron visibilizar nuevas amenazas y retos para la seguridad. Al respecto, Buzan establece que el sistema de seguridad posterior al fin de la era bipolar está caracterizado por un proceso de ampliación y profundización del concepto de seguridad en el cual el Estado ya no es el único referente para explicar una realidad cada vez más compleja (Buzan, Waever y De Wilde, 1998).

Así, este enfoque sugiere que los debates sobre la seguridad internacional estudian el sistema internacional y el individuo y el papel que desempeñan las organizaciones internaciones, los gobiernos locales y regionales, las organizaciones no gubernamentales, la opinión pública e, incluso las fuerzas del mercado global (Bassedas, 2012). Sin embargo, en contrapartida, tampoco se puede desconocer que la falta de un orden en el sistema internacional acarrea una falta de consenso para allanar el camino hacia un marco de compresión totalizante de las amenazas que afectan seguridad.

4 Varios autores sostienen la tesis, argumentada con base en la teoría de las transiciones hegemónicas, que el poder de Estados Unidos atraviesa por una fase de declive como potencia. 


\section{Estados Unidos}

Contrario a la opinión de los analistas escépticos, Estados Unidos continúa siendo un actor determinante en la dinámica del sistema internacional contemporáneo y en la seguridad mundial. Su poder de influencia en la política mundial no ha sido diezmado totalmente: desde el ámbito económico, aunque ha mostrado desequilibrios con una deuda pública que supera el $100 \%$ de su PIB, aun muestra superioridad indiscutible como potencia capitalista. Además, aunque desde 2007 China es la principal productora de software y hardware, el $84 \%$ de las ganancias de este rubro sigue estando en manos de capitalistas estadounidenses, y lo mismo sucede en el ámbito especulativo donde las ganancias por servicios financieros han pasado del $47 \%$, de 2007 , al $66 \%$, en 2013 (Senado de México, 2014). Igualmente, un $45 \%$ de las 500 principales empresas transnacionales son de capital estadounidense, así como una buena parte de los medios de comunicación con más impacto mediático en la población mundial.

Sin embargo, hay que matizar esta posición. A pesar del poder de Estados Unidos, su transición hacia la hegemonía en el sistema internacional contemporáneo (Brzeziński, 1997; Fukuyama, 1995) no ha logrado consolidarse. Desde la década de los años noventa, la capacidad de respuesta por parte de la hiperpotencia ante los conflictos regionales que amenazaban la estabilidad de la seguridad mundial fueron bastante bajas. Cabe recordar que el genocidio de Ruanda recibió poca atención por parte de la ONU, mientras que los ojos del mundo posaban su mirada al conflicto de los Balcanes de mayor interés de Estados Unidos (Melven, 2007). Este caso sirve de argumento teórico para establecer cómo se produce el declive de las potencias hegemónicas; "aunque descubren que siguen contando con mayores capacidades de poder en el sistema internacional, al mismo tiempo estas se ven 'agobiadas' por una 'sub-limitación nacional' [...] y por la sobre expansión imperial que permite el ascenso de otros grandes poderes” (Ghotme, 2010, p 49).

De esta manera, la tendencia hacia la multipolaridad encuadra en el periodo de transición hacia un nuevo orden internacional, con las problemáticas y las oportunidades que esto supone. Es posible que en 
los próximos años se consolide el sistema internacional contemporáneo como una red de intercambios interdependientes, basado en un equilibrio de poder distinto a la hegemonía de los poderes europeos del siglo XIX, y al bipolarismo de los años posteriores a 1945. En este nuevo escenario, los poderes emergentes desempeñarán un papel protagonista en estas relaciones internacionales, como es el caso de Brasil en el nivel hemisférico, toda vez que América Latina aparece con más autonomía dentro de este contexto (De Onis, 2008).

La tendencia hacia el multipolarismo ha atizado el debate sobre el declive de la potencia hegemónica. De hecho, el declive de Estados Unidos en las relaciones interamericanas - bastión por excelencia de su dominio-, ha sido un argumento poco explorado desde la academia como tema de debate del declive de la potencia. Sin embargo, algunos analistas han establecido consenso sobre la idea de la muerte de la Doctrina Monroe como política de dominio en la región, tal como lo cita Drekonja-Kornat (2011) del Task Force Report sobre Latinoamérica patrocinado por el Council on Foreing Relations: " $\mathrm{Si}$ alguna vez existió una era de hegemonía estadounidense en América Latina, se terminó" (Council on Foreing Relations, 2008). ${ }^{5}$ De este modo, la esfera de influencia de Estados Unidos en América Latina, comúnmente denominada como el patio trasero, ganó un margen de maniobra para convertirse en un actor con mayor influencia en el sistema internacional contemporáneo. ${ }^{6}$

5 Desde la llegada al poder del presidente Hugo Chávez (1999-2013) en Venezuela con una línea de política exterior anti hegemónica, emergió un nuevo modelo de integración regional en respuesta a la desgatada Organización de Estados Americanos. La integración económica propuesta desde Washington basada en un área de Libre Comercio en las Américas (ALCA), en los años noventa, fue rechazada por movimientos sectores locales, y la región abrió paso a una integración con características más simétricas a las que ofrecía el modelo del ALCA.

6 El hemisférico americano en la actualidad comparte al menos dos modelos de regionalismo antagónicos. Por una parte, Ecuador y Bolivia integraron el proyecto de Venezuela y Cuba, de la Alianza Bolivariana para los Pueblos de Nuestra América - Tratado de Comercio de los Pueblos (ALBA), mientras que por otra Colombia, Perú y Chile afianzaron su relación con Estados Unidos mediante la firma de los Tratados de Libre Comercio (TLC) bilaterales que remplazaron la propuesta inicial del Estados Unidos para establecer el ALCA. 
El descuido de su zona de influencia hemisférica, refuerza la visión de que las acciones exteriores de Estados Unidos se centraron en la guerra contra el terrorismo después de los atentados del 11 de septiembre, lo cual, a su vez, permitió una reformulación de la agenda en los estudios de la seguridad internacional (Buzan y Hansen, 2009). La autodefensa (unilateralismo) y los ataques preventivos adaptados para combatir las amenazas terroristas que amenazan la estabilidad internacional (United States, 2002) contrastaron con el enfoque de seguridad multilateral en favor de la defensa del individuo que surgió en el nuevo sistema internacional, con un retorno marcado al concepto de la seguridad nacional en detrimento de otros enfoques de seguridad.

\section{Los años de la contención y la Posguerra Fría}

Las consecuencias del fin de la Guerra Fría en la formulación de la estrategia de seguridad de Estados Unidos, en relación con los desafíos de un nuevo orden internacional fueron evidentes. Antes de la caída del comunismo, estrategia de seguridad y defensa estadounidense concentró sus esfuerzos en el enfrentamiento con la Unión de Repúblicas Socialistas Soviéticas (URSS) mediante dos doctrinas, la primera la disuasión nuclear, formulada como mecanismo de prevención de ataques nucleares por parte de la Unión Soviética, y la segunda, la contención, cuyo objetivo era impedir que la este país ampliara su espectro de influencia basada en el comunismo (Cidob , 2009). Sin embargo, la Guerra del Golfo (1990-1991) fue uno de los primeros acontecimientos que determinaron la política de seguridad y defensa norteamericana durante los años noventa, en la medida en que la disuasión no resultó ser un método efectivo por las características de sus nuevos rivales en Medio Oriente. Para entonces, Irak y el desafío fundamentalista iraní podrían proporcionar armas de destrucción masiva a los grupos terroristas.

Sin embargo, la National Military Strategy de 1992 adicionó, al menos, tres principios la estrategia de defensa estadounidense. El primero, la presencia avanzada que aseguraba el desarrollo de un anillo 
de seguridad por el mundo, el segundo, respuesta a la crisis, que aseguraba una rápida reacción a la más de una crisis regional, en sintonía con su posición privilegiada en el sistema internacional, y finalmente, desarrollar capacidad de reconstrucción militar (Cidob , 2009). Las estrategias posteriores de la década de los años noventa incorporaron algunos elementos propios de la etapa de la Posguerra Fría, como el mantenimiento de la paz mediante el envío de misiones humanitarias (National Military Strategy, 1995) y en 1997 disuasión con actividades en tiempos de paz y liderazgo activos de las alianzas.

Desde el plano de la seguridad humana, el gobierno del presidente Bill Clinton hizo parte de del protocolo de Kioto para la reducción de gases en diciembre de 1997 (Saura Estapà, 2003). De esta manera, Estados Unidos accedió a participar en la firma de un instrumento jurídico capaz de garantizar la sostenibilidad medioambiental como un eje propuesto en la ampliación de las amenazas tradicionales que abarcan el enfoque de la seguridad humana (libertad ante la necesidad). Sin embargo, con los ataques de las Torres Gemelas en septiembre de 2001 se redefinió el contexto general de su política de seguridad para hacer frente a las amenazas que atentaron directamente su seguridad nacional.

\section{¿El fin de la hegemonía? Los atentados del 9/11}

Los ataques del 9/11, redefinieron el panorama de la seguridad mundial y el papel de Estados Unidos como la potencia líder garante del mantenimiento de la paz en el sistema internacional. El equilibrio del poder se desestabilizó nuevamente de forma similar al siglo anterior. En este nuevo contexto, Estados Unidos recibió, en principio, el apoyo de un número considerable de miembros de la comunidad internacional para hacer frente a la nueva amenaza que fue denominada por la Doctrina Bush como el Eje del mal. De esta forma, el gobierno de Washington replanteaba la estrategia de seguridad hasta entonces definidas para hacer frente a las amenazas tradicionales de tipo convencional propias de la Guerra Fría como las de tipo nuclear. Ahora el terrorismo, global, junto con las armas de destrucción masiva fueron añadidos al complejo de seguridad tradicional, convirtiendo el 
radicalismo y la tecnología en las dos principales amenazas para la nación americana (Cidob, 2009).

Dos elementos notorios en el cambio de visión de la política de seguridad de Estados Unidos después de los ataques del 9/11, derivaron de la Operación Libertad Duradera en Afganistán (2001). El retorno a la defensa de la seguridad nacional como política del gobierno republicano del presidente George W Bush (2002-2010) estableció los conceptos de la autodefensa y la acción preventiva (United States, 2002). En adelante hasta la llegada del gobierno del presidente Barack Obama (2010-2016) persistió en la política exterior estadounidense el ideal de combatir el terrorismo para extender la paz, instigando sociedades libres, abiertas y democráticas en el mundo, tal como se muestra a continuación (tabla 1).

Tabla 1. Evolución de la doctrina de seguridad

estadounidense después del 9/11

\begin{tabular}{|c|c|c|c|c|}
\hline $\begin{array}{l}\text { Prioridades en } \\
\text { Seguridad y } \\
\text { Defensa Obama }\end{array}$ & $\begin{array}{c}\text { Estrategia de } \\
\text { seguridad, } 2006\end{array}$ & $\begin{array}{c}\text { Estrategia de } \\
\text { seguridad, } 2002\end{array}$ & Doctrina Bush & \\
\hline $\begin{array}{l}\text { Respaldo por } \\
\text { parte de la } \\
\text { comunidad } \\
\text { internacional. }\end{array}$ & $\begin{array}{l}\text { Apoyo } \\
\text { restringido de } \\
\text { la sociedad } \\
\text { internacional. }\end{array}$ & $\begin{array}{l}\text { Apoyo } \\
\text { restringido de } \\
\text { la sociedad } \\
\text { internacional. }\end{array}$ & $\begin{array}{l}\text { Respaldo por } \\
\text { parte de la } \\
\text { comunidad } \\
\text { internacional. }\end{array}$ & Apoyos \\
\hline $\begin{array}{l}\text { Grupos } \\
\text { terroristas. }\end{array}$ & $\begin{array}{l}\text { Terrorismo } \\
\text { de Estados } \\
\text { antidemocráticos. }\end{array}$ & $\begin{array}{l}\text { Las armas de } \\
\text { destrucción } \\
\text { masiva } \\
\text { (tecnología), y el } \\
\text { radicalismo. }\end{array}$ & $\begin{array}{l}\text { Seguridad } \\
\text { bidimensional } \\
\text { convencional/ } \\
\text { nuclear obsoleta. } \\
\text { Terrorismo }\end{array}$ & $\begin{array}{l}\text { Definición } \\
\text { de la } \\
\text { amenaza. }\end{array}$ \\
\hline $\begin{array}{l}\text { Al Qaeda, } \\
\text { Estado Islámico. }\end{array}$ & $\begin{array}{l}\text { Estados canallas } \\
\text { (Irán y Siria). }\end{array}$ & $\begin{array}{l}\text { El Eje del mal } \\
\text { Estados Canallas: } \\
\text { Irak (antes del } \\
\text { 2003) y Corea } \\
\text { del Norte. }\end{array}$ & $\begin{array}{l}\text { Transformación } \\
\text { de la amenaza de } \\
\text { China y Rusia... }\end{array}$ & $\begin{array}{l}\text { Enemigos } \\
\text { definidos. }\end{array}$ \\
\hline $\begin{array}{l}\text { Retirada de } \\
\text { las tropas de } \\
\text { combate de } \\
\text { Irak. Ayuda } \\
\text { diplomática. }\end{array}$ & $\begin{array}{l}\text { Ataques } \\
\text { ofensivos, con } \\
\text { defensas activas, } \\
\text { infraestructura } \\
\text { de respuesta y } \\
\text { planificación de } \\
\text { inteligencia. }\end{array}$ & $\begin{array}{l}\text { autodefensa } \\
\text { (unilateralismo) } \\
\text { y la acción } \\
\text { preventiva. }\end{array}$ & $\begin{array}{l}\text { Guerras } \\
\text { interestatales. }\end{array}$ & Medios \\
\hline
\end{tabular}

Fuente: elaboración propia a partir de Cidob (2009) y Bejer (2005). 
La tabla 1 muestra cómo las amenazas en el sistema internacional contemporáneo se han trasformado sustancialmente. El mecanismo de balance de poder instituido desde Westfalia, para algunos, resultaría obsoleto en la medida en que los grupos terroristas — como actores no estatales- muestran más disposición a asumir los riesgos de usar armas de destrucción masiva. En consecuencia, la doctrina de disuasión, empleada por Estados Unidos en la era de la Guerra Fría no funciona con la misma eficacia que con la Unión Soviética. De esta forma, desde el punto de la seguridad internacional, cabría preguntar si en el actual des-orden mundial del sistema internacional contemporáneo ¿̇existe un desdibujamiento del papel de los Estados como actor principal de las relaciones internacionales?; de ser positiva esta afirmación y en relación con Estados Unidos ¿la crisis del Estado moderno refuerza la hipótesis del declive de Estados Unidos como potencia hegemónica?

Jesper Bejer (2005) aborda el problema desde la teoría de las Relaciones Internacionales. Este autor se apoya en los planteamientos de Waltz (2002), en cuanto a que los Estados continúan siendo los protagonistas del sistema internacional en la medida en que las leyes fundamentales de los Estados siguen siendo iguales. Además, las condiciones en que opera el terrorismo transnacional (las redes de terrorismo), deben necesariamente contar con un punto central ya que están ancladas en un territorio determinado — generalmente en Estados frágiles- desde donde opera su logística y sus recursos; en otras palabras, para que una estructura terrorista sobreviva debe habitar en un espacio seguro central. Esta suerte de dependencia estatal alentó la política de seguridad estadounidense que fijó la lucha contra los Estados canallas (Irak, Irán, Corea del Norte y Siria entre otros).

En síntesis, cabe destacar que la hegemonía estadounidense se vio opacada por la creciente multipolaridad del sistema internacional contemporáneo. Un análisis al desarrollo de la política de defensa de Estados Unidos en las últimas décadas deja ver que difícilmente se pueden desplegar acciones consistentes en diferentes partes con el fin de afianzar una hegemonía global; sin embargo, es prematuro afirmar que existe una crisis del modelo del Estado westfaliano en el mundo. Las leyes formuladas en la escuela neorrealista aún privilegian las acciones de los Estados, en caso de la lucha contra el terrorismo. 


\section{Rusia}

En el sistema internacional contemporáneo, Rusia empieza a emerger con mayor fuerza en la política internacional, tratando de volver a ser lo que en un pasado fue: la Gran Potencia. El presidente Vladimir Putin intenta devolverle a Rusia la imagen de la madre patria logrado en su plenitud durante el periodo de la Segunda Guerra Mundial con el Ejército Rojo a la cabeza, e igualmente impulsar el prestigio internacional de Rusia. Un análisis de la política exterior rusa, basado en la estrategia de seguridad nacional y los discursos en diferentes escenarios internacionales de Putin, permiten entender el concepto de seguridad en las relaciones internacionales contemporáneas. Este aparte tratará, en primer lugar, la seguridad de la Federación Rusa, y en segundo aspecto, el nuevo concepto de seguridad en el discurso y las acciones del Estado ruso. Finalmente, se concluye que la seguridad de Rusia se interpreta en un concepto clásico en las relaciones internacionales, a pesar de que los nuevos análisis de la seguridad internacional se verán reflejados y demandados desde el Estado.

\section{La Seguridad en la Federación de Rusia}

Durante el siglo XX, el sistema internacional tuvo cambios y episodios que modificaron la estructura de poder y que generaron diferentes consecuencias, marcadas con las guerras mundiales y diferentes conflictos que se originaron por la necesidad de diversos Estados de conseguir poder y posición en el nivel global, con el fin de mejorar sus capacidades y beneficios. Sin embargo, con el fin de la Guerra Fría y la transición al sistema de relaciones actual, Rusia trata de mantener un estatus como potencia, pero a la vez, se observa un Estado inmerso en discursos y dinámicas occidentales, es decir, Rusia no volvería a visualizarse como un Estado política y económicamente comunista.

De esta manera, Rusia de Putin entró en el siglo XXI al escenario internacional en tres aspectos importantes. Primero, Rusia implementaría un discurso y un actuar con comportamientos occidentales 
(entrada a la Organización Mundial del Comercio (OMC), respeto por el Derecho Internacional, relaciones capitalistas tanto en occidente como sus vecinos orientales); Segundo, la cohesión social o unidad del pueblo ruso a través del resurgimiento del sentir nacionalista exsoviético de potencia global y prestigio imperial, y por último, la manifestación y defensa en su política exterior de un mundo multipolar.

Esto trae consecuencias en la concepción de seguridad de Rusia y en su política exterior al postular sus intereses vitales como: la defensa del Estado, entendida como el mantenimiento de la integridad territorial del Estado ruso, para garantizar su viabilidad económica y política; potencializar el poder del Estado en el plano externo como potencia y ser reconocido por los grandes polos de poder, como importante y decisorio en la estructura de poder del sistema internacional, que le permitirá mantener el statu quo del sistema internacional multipolar al defender y ejecutar un equilibrio de poder para impedir una manifestación por parte de Estados Unidos como potencia unipolar y así mismo acercarse a Estados occidentales que en un momento lo seguían viendo como Estado comunista y enemigo; y por último, la consolidación de un espacio geopolítico en Asia Central y Estados satélites ex-soviéticos, que le permiten consolidarse como potencia regional, ese espacio geopolítico es denominado extranjero cercano. ${ }^{7}$

Teniendo en cuenta que el concepto de seguridad igualmente cambió después de la Guerra Fría, cuando la mirada político-militar se subestima y sobrepasa a una mirada multidimensional de la seguridad, y la atención se centra en el individuo, dando origen a la seguridad humana, que interactúa con otros actores no estatales, la seguridad seguirá siendo implementada — sea seguridad humana o no- por el Estado. Así, al estudiar la Estrategia de Seguridad Nacional de Rusia (ESNR)

7 El extranjero cercano es ese espacio antiguo soviético donde viven más de 20 millones de rusos. Es importante que, en los Estados de Armenia, Azerbaiyán, Bielorrusia, Georgia, Kazajistán, Kirguistán, Moldavia, Tayikistán y Ucrania, Rusia mantiene bases militares activas junto con un proyecto de integración económica que se creó en el 2000, denominado la Comunicad Económica Euroasiática. Así mismo, Putin recuperó de forma ecléctica los símbolos del pasado (el águila zarista y la música del himno soviético entre ellos) (Facio, 2015). 
del 31 de diciembre de $2015,{ }^{8}$ se evidencia el concepto clásico del realismo, que postula el protagonismo del Estado en el monopolio del uso de la fuerza. Por lo tanto, la seguridad nacional se estructura desde el Estado como el principal actor de la seguridad de sus ciudadanos, pero también lo militar es el principal factor al dentro y fuera del Estado.

Sin embargo, aunque el discurso global esté mostrando la seguridad humana fuera de la ratio del Estado, el concepto de seguridad de Rusia debe ser entendido como la protección del individuo y la sociedad (seguridad humana) de aquellos peligros que emanan de las amenazas internas y externas, percibidas por el mismo Estado. Al respecto, la seguridad nacional incluye la defensa de la Federación Rusa y de todos los tipos de seguridad previstos por la Constitución del Estado —estatal, pública, individual, de información, ambiental, económica, transporte y energética-. Es decir, como la mayor parte de las grandes potencias contemporáneas, adopta un amplio enfoque multidisciplinar, teniendo en consideración el conjunto de amenazas, riesgos y la propia concepción estratégica rusa. El documento confirma además la interconexión existente entre la seguridad nacional y el desarrollo socioeconómico del país, que lleva a deducir que la seguridad humana es exigida y sostenida en el Estado, no por otros actores no estatales como lo sostendrían los neoliberales institucionales.

Al comparar las ESNR de los años 2009 y 2015, se muestran elementos que, a priori, no están inmersos dentro de factores de seguridad nacional del Estado como la ecología, sin embargo, estos se ven inmersos y defendidos por este, como se muestra a continuación en la siguiente tabla:

8 "el papel de la fuerza como uno de los factores de las relaciones internacionales no está en declive [...] la disuasión estratégica y la prevención de conflictos militares se lleva a cabo a través de la preservación del potencial de disuasión estratégica a un nivel suficiente y garantizando el nivel específico de preparación para el combate de las Fuerzas Armadas de la Federación de Rusia”. Véase en Documento en la página web del Kremlin. http://en.kremlin. ru/events/president/news/51129 
Tabla 2. Comparativo de las estrategias de seguridad nacional de Rusia

\begin{tabular}{|l|l|}
\hline \multicolumn{1}{|c|}{ ESNR 2015} & \multicolumn{1}{|c|}{ ESNR 2009 } \\
\hline $\begin{array}{l}\text { El papel de la fuerza como un factor en } \\
\text { las relaciones internacionales no está } \\
\text { disminuyendo, en defensa del país. }\end{array}$ & $\begin{array}{l}\text { La aplicación de fuerza militar para la } \\
\text { protección de los intereses nacionales es } \\
\text { posible. }\end{array}$ \\
\hline $\begin{array}{l}\text { Seguridad del Estado y de la sociedad. } \\
\text { la estabilidad del sistema de relaciones } \\
\text { económicas internacionales. El Estado } \\
\text { como garante de la seguridad de la } \\
\text { persona y los derechos de propiedad. }\end{array}$ & $\begin{array}{l}\text { "Investigación, tecnología y educación”, } \\
\text { "Estabilidad estratégica y a alianzas en } \\
\text { condiciones de igualdad”. }\end{array}$ \\
\hline $\begin{array}{l}\text { Elevación de calidad de vida de los } \\
\text { ciudadanos. Fortalecer la cohesión social. }\end{array}$ & $\begin{array}{l}\text { Crecimiento económico. Fortalecimiento de } \\
\text { la unidad interna. Cohesión social, poder } \\
\text { intangible del Estado. }\end{array}$ \\
\hline $\begin{array}{l}\text { Salud, cultura, ecología, seguridad } \\
\text { alimentaria. }\end{array}$ & $\begin{array}{l}\text { Cooperación entre Estados, solución pacífica } \\
\text { de controversias. }\end{array}$ \\
\hline $\begin{array}{l}\text { La Federación de Rusia construye } \\
\text { sus relaciones internacionales en los } \\
\text { principios del derecho internacional, para } \\
\text { desarrollar relaciones con la OTAN sobre } \\
\text { la base de la igualdad. }\end{array}$ & No tolerará la ampliación de la OTAN. \\
\hline Ártico y “extranjero cercano". & Priorizar intereses y su política en el Ártico \\
\hline
\end{tabular}

Fuente: elaboración propia.

ESNR 2015: documento completo en http://mepoforum.sk/wp-content/uploads/2015/08/NDSRF-2009-en.pdf

ESNR 2009: documento en la página web del Kremlin. http://en.kremlin.ru/events/president/ news/51129

La comparación demuestra, igualmente, que la seguridad abarca una multidimensionalidad, sujeta a la institucionalidad del Estado, por ello, no podría afirmarse una crisis del Estado-nación cuando en la era de la globalización, hipermodernidad ${ }^{9}$ y el desdibujamiento de las fronteras. Por el contrario, el Estado sigue moldeándose y reestructurando su poderío militar para contrarrestar nuevas amenazas que

9 El hipermodernismo Lipovestsky lo define como un proceso de personalización, destrucción de las estructuras colectivas de sentido, hedonismo, consumismo, tensiones paradójicas en los individuos y en la sociedad civil, la seducción como forma de regulación social, rechazo de la violencia política y aumento de la consideración ciudadana de los valores de la democracia. Es decir, es el individualismo lo que impera, aun en la misma estructura del Estado (Lipovetsky, 2006). 
encierran la seguridad humana. Por lo mismo, de aquí parte la necesidad de formular el estudio de la seguridad desde el Estado, en detrimento de las nuevas definiciones conceptuales o interpretaciones, porque el individuo, finalmente, se encuentra sujeto de una u otra manera a esa noción de Estado y Estado-nación. Pareciese entonces que la seguridad en las relaciones internacionales sobrevive en cuanto a la misma supervivencia del Estado, como lo demuestra la ESNR.

\section{¿Nuevo concepto de seguridad? Discursos y acciones en el Estado ruso}

En su discurso a la Conferencia de Seguridad de Munich, en el 2007, Putin sostenía que, la guerra entre Estados es una realidad en el sistema internacional, y por ello debe usarse mecanismos jurídicos como la carta de la ONU. Así mismo, lo reiteró en el discurso del 70 aniversario de la Asamblea General de la ONU de 2015, en el cual textualmente afirmó:

En los 70 años de las Naciones Unidas han existido diferencia en el Consejo de Seguridad y han recurrido al veto, siendo natural para una organización. La fortaleza está en la pluralidad, y las decisiones vienen de resoluciones. Cualquier medida que tome un Estado contraria a dichas resoluciones son ilegitimas y desafían el derecho internacional. (Putin, 2015)

Rusia se define como un Estado que defiende el derecho internacional, los mecanismos pacíficos de solución de controversias, pero que, impulsado por la defensa de sus intereses, se ve obligado a usar la fuerza. Aun existiendo grupos privados, no estales que compiten por el monopolio del uso de la fuerza, el Estado sigue proyectado lo que percibe y encuentra en la realidad y lo real ${ }^{10}$ amenazas para el individuo. En la conferencia Club Internacional de Debates Valdai en octubre de

10 Lo real hace referencia a Slavoj Zizek. Este autor interpreta lo real, como una realidad sin serlo (Zizek, 2002). Un ejemplo del concepto es la realidad virtual, donde el individuo la toma como realidad cuando no lo es en su forma tangible. De una manera homologa ocurre cuando el Estado percibe amenazas de lo real, en una realidad material que experimenta, pero es una realidad 
2015, Putin expuso que nos encontramos en un escenario en que la realidad no se encuentra descrita como lo plantea el discurso hegemónico occidental en la actualidad: entre buenos y malos, entre democracia y fundamentalismo, entre seguridad global y terrorismo, sino que todo hace parte de la misma manifestación ideológica del capitalismo. También afirma que, desde Estados Unidos, hasta los neonazis y los radicales islámicos desestabilizan el orden mundial queriéndolo rehacer a su medida; por ende, la política exterior de Rusia se enfoca en la defensa del multilateralismo, como la mejor opción de surgimiento de potencia global.

Y aunque Rusia no pretende ir en contra de esa manifestación ideológica del capitalismo, lo que pretende es mantener un equilibrio de poder que fomente seguridad, empezando desde el desarrollo económico ${ }^{11}$ y calidad de vida del individuo. Igualmente, la estrategia de seguridad nacional de Rusia 2015 se basa en discursos, actuaciones y políticas occidentales, siendo la manifestación ideológica e irreal del capitalismo, y es irreal porque ya no se encuentra en el discurso hegemónico (o estadounidense). Por lo tanto, la estrategia de seguridad para Putin no está encerrada entre buenos y malos o fundamentalistas o capitalistas, sino al individuo sumergido en el discurso posmoderno de seguridad, es decir, ideológicamente capitalista. La seguridad en las relaciones contemporáneas es: discursivamente en la lógica capitalista (cooperación internacional, derechos humanos, terrorismo, premisas idealistas de utilización de mecanismo de solución pacífica de controversias) y a su vez el mantenimiento de la multipolaridad del sistema internacional, como si esta condición amparara los aspectos más relevantes de la Estrategia de Seguridad de Rusia del 31 de diciembre de 2015.

virtual, algo que se dice está presente, pero no es posible tocarla, por ejemplo, la muerte. Así lo es la seguridad humana para el Estado.

11 La teoría del Realismo Periférico, de Carlos Escudé (2012), indica que los Estados débiles deben buscar un desarrollo económico y riqueza para el Estado, mas no una preocupación por la seguridad del Estado, plantea desde entonces el bienestar ciudadano en términos económicos que a su vez les brindaría una estabilidad a sus ciudadanos y también al sistema internacional. Desde esa perspectiva, se puede interpretar la política exterior rusa desde el marco del realismo periférico. 


\section{La seguridad humana y el Estado}

La seguridad humana podría interpretarse como el individuo en el centro de la seguridad y la seguridad como derecho humano. Al postular la vida como el factor más importante de la libertad ante el miedo, la seguridad no sería otra cuestión que la vida, ¿ por qué hablar de actores diferentes al Estado que pretenden sobrellevar la seguridad humana y convertir al Estado como un elemento irrelevante y no indispensable en el objetivo de la seguridad internacional? Para Strange (2001), el Estado se retiró, mas no desapareció. Podría decirse los Estados se acomodan a las dinámicas coyunturales de la sociedad mundial, y para ello el ejemplo ruso es la forma de palpar que la seguridad es esencial y más cuando los centros de poder se diversifican (Quintero y Fula, 2016).

Bajo estas circunstancias, cabe indicar que, aunque el Estado de derecho es una conquista occidental, la historia muestra que no todos los Estados son iguales en sus formas de relacionarse con los demás agentes internacionales. Esto debido a que cada caso en particular define cuáles son los temas relevantes para tratar durante una propuesta de política exterior; esto teniendo en cuenta que en Rusia la estrategia de Seguridad Nacional se encuentra en términos económicos e intercambio de información, para sobrellevar el avance económico y bienestar de la calidad de vida de sus ciudadanos. La seguridad entonces está inmersa en la misma concepción de Estado moderno y a la lógica de supervivencia de este, aunque se encuentre individualizada actualmente la seguridad internacional, el individuo se somete y se sujeta a las relaciones estatales como una fuerza centrípeta.

Por último, al analizar la concepción de Rusia como Estado en el siglo XXI, el fundamentalismo, el terrorismo, la democracia, la multiculturalidad, derechos humanos, medioambiente, conceptos que aparentemente se encuentran en orillas distintas, hacen parte de un mismo esquema mental: seguridad internacional. La seguridad contemporáneamente es entendida como modelo de pensamientos que se construyen las concepciones de "amigo-enemigo", como una condición de pensamiento alienante, sin posibilidad de crítica, pero queriendo ser encontrada la seguridad en su plenitud y reflejada en la seguridad humana. 


\section{Conclusiones generales}

La seguridad en las relaciones en el sistema internacional contemporáneo, no es solo económica y un exclusivo enfoque en el individuo, como los dirían los teóricos críticos de los enfoques clásicos. Aun cuando, se llevó a cabo una reformulación en las doctrinas de seguridad (el caso de Estados Unidos en los años noventa) para responder a nuevos restos de la Posguerra Fría, la coyuntura del 9/11 obligó a retornar a los enfoques clásicos de la seguridad. Esta política se reflejó en los documentos de seguridad posteriores.

Igualmente, la seguridad en el sistema internacional contemporáneo no muestra un avance en la praxis. El análisis a las estrategias de seguridad nacional de Rusia y Estados Unidos evidencia que: 1) el uso de la fuerza continúa como el factor principal frente a las amenazas 2) los enfoques teóricos clásicos como el neorrealismo continúan vigentes para interpretar la realidad internacional, donde se privilegia la acción del Estado como el actor principal del sistema internacional.

Cabe establecer que, la crisis del Estado-nación y su ineficiencia para garantizar la seguridad de los individuos no es otra cuestión que un mero pretexto de lo real; es decir, no se puede negar que la seguridad sigue siendo clásica y tratar de transformarla por una hipermodernidad que, aunque individual en aspectos económicos y comerciales el amparo de sentirse seguro y protegido, es una labor exclusiva y esencial del Estado.

El fin de la Guerra Fría permitió exponer y aplicar la seguridad humana aun por fuera de la concepción del Estado, como una necesidad para instaurar la paz internacional, dando cabida a nuevos argumentos como los de los securistas críticos que pretendían entender al Estado como el instrumento generador de inseguridad. En ese sentido, la seguridad quiso ser comprendida en el individuo como el centro, generando un abanico de definiciones de seguridad. Sin embargo, más allá de definirla, la cuestión central radica en dónde encontrarla y demandarla; por esto, el Estado se vio condicionado, pero fue evolucionando a la vez como el garante de la seguridad del individuo. Así, al analizar las estrategias de las grandes potencias actuales, vemos como la seguridad nacional vuelve a ponerse en la punta de lanza de 
las relaciones internacionales, donde esta absorbe la seguridad humana y su multidimensionalidad.

\section{Referencias}

Ariza, N. (2010). La aplicabilidad del concepto de seguridad humana en América Latina y el Caribe: El desarrollo humano como fuente de seguridad. OASIS, 15.

Arrighi, G. y Beverly, S. (2001). Caos y orden en el sistema mundo moderno. Madrid: Akal.

Bassedas, M. (2012). La Seguridad Internacional después de la Guerra Fría ¿Avanzando hacia una nueva doctrina de seguridad humana? Revista CIDOB d'Afers Internacionals, 100. p, 2

Bejer, J. (2005). 11 de septiembre y la continuidad del Sistema Internacional. Trabajos y ensayos, 1-16.

Brzezi冈ski, Z. (1997). El Gran Tablero Mundial: La supremacía estadounidense y sus imperativos estratégicos. (M. Salomón, Trad.) Barcelona: Paidós.

Buzan, B. y Hansen, L. (2009). The Evolution of International Security Studies. Londres: Cambridge University Press.

Buzan, B., Waever, O. y De Wilde, J. (1998). Security: A New Framework for Analysis. London: Lynne Rienner Publishers.

CIDOB (2009). La estrategia de defensa y seguridad de EEUU desde la Segunda Guerra Mundial. Madrid. CIDOB d'Afers Internacionals

Council on Foreing Relations . (2008). US-Latin American Relations: A New Direction for a New Reality. Nueva York.

De Onis, J. (2008, nov.-dic.). Brazil’s Big Moment. Foreing Affairs, 10-123.

Drekonja-Konat, G. (2011). El ocaso de la Doctrina Monroe. En D. Wollarad, G. Gihold, y M. Mols, La agenda internacional de América Latina. Entre nuevas y viejas alianzas. Buenos Aires: Nueva Sociedad.

Escudé, C. (2012). Principios de realismo periférico: vigencia de una teoría argentina ante el ascenso de China. Buenos Aires: Ed. Lumière.

Facio, H. (2015). Rusia, de los zares a Putin (1880-2015). Bogotá: Uniandes .

Fukuyama, F. (1995). El fin de la historia y el último hombre. Madrid: Planeta-Agostini. 
Ghotme, R. (2006). Orden, des-orden y antiorden mundial. Problemas en las Relaciones Internacionales. Revista de Relaciones Internacionales, Estrategia y Seguridad, 1 (2).75-103

Ghotme, R. (2010). La configuración del poder en el sistema internacional contemporáneo. Revista de Relaciones Internacionales, Estrategía y Seguridad, 6 (1), 49.

Graham, J. W. ((abril-junio) de 2005). La OEA se hunde: ¿Merece ser salvada? Foreing Affairs.

HIrsl, M. (2011). Las relaciones entre América Latina y Estados Unidos en los tiempos de la Post-Guerra Fría. En D. Wollarad, G. Maihold y M. Mols, La Agenda Internacional de América Latina; Entre nuevas y viejas alianzas (p. 32). Buenos Aires : Nueva Sociedad; Fundación Friedrich Ebert.

Immanuel, W. (2005). La Decadencia del Imperio : Estados Unidos en el Mundo Caotico . Madrid: Txalaparta.

Kennedy, P. (1988). Auge y caída de las grandes potencias. Barcelona: Plaza y Janes.

Histórico discurso de Valdimir Putin en la 70 Asamblea General de la ONU (28 de 09 de 2015). Recuperado el 16 de julio de 2016, https://www. youtube.com/watch?v=2ioGWPJuyW8

Iglesias, M. (2016, jul.). La estrategia de seguridad nacional de la Federación Rusa. Recuperado de http://www.ieee.es/Galerias/fichero/docs_opinion/2016/DIEEEO25-2016_ESN_Rusia_MLI.pdf

Lipovetsky, G. (2006, jul.). Los tiempos hipermodernos. (A. P. Moya, Trad.). Barcelona: Anagrama. Recuperado de El Cultural.com .

Melven, L. (2007). Un pueblo traicionado: el papel de occidente en el genocidio de Ruanda. Barcelona: Intermón Oxfam.

Ohmae, K. (1997). El fin del Estado-Nación. El ascenso de las Economías Regionales. Santiago de Chile: Andrés Bello .

Pastrana, E., Vélez, R. y Castro, R. (2014). Colombia y la alianza del Pacífico: un proyecto regional de cara a la multipolaridad creciente. En E. Pastrana y H. Gehring, Alianza del Pacífico Mitos y Realidades (p. 174). Bogotá: Universidad Santiago de Cali.

Pearson, F. y Rochester, M. (2000). Relaciones Internacionales (4 $\left.{ }^{\mathrm{a}} \mathrm{ed}.\right)$. Bogotá: McGraw-Hill.

Pérez de Armiño, K. (2006). El concepto y el uso de la seguridad humana: análisis crítico de sus riesgos y potencialidades. Revista CIDOB d'Afers Internacionals, 76. 
Putin interviene en el Club Internacionla de Debates Valdai. (22 de 10 de 2015). Youtube. (RT). Recuperado el 16 de julio de 2016, de https:// www.youtube.com/watch?v=G0XX1Dtj3h8

Putin, C. (2007). Putin's memorable Munich Speech 2007. Youtube. (RussianPerspective) Recuperado el 15 de noviembre de 2016, de https:// www.youtube.com/watch?v=hQ58Yv6kP44

Quintero, S. y Fula, A. (2016). Política exterior rusa: acciones en Europa y América Latina. Artículo no publicado. Bogotá.

Saura Estapà, J. (2003). El cumplimiento del Protocolo de Kioto sobre cambio climático. Barcelona: Publicaciones de la Univeridad de Barcelona.

Senado de México. (2014). Los retos actuales de la izquierda en América Latina y el proyecto de dominación de Estados Unidos. México: Grupo de Investigación sobre América del Norte del Centro de Estudios Hemisféricos y sobre Estados Unidos (Cehseu).

Strange, S. (2001). La retirada del Estado: la difusión del poder en la económica mundial. Barcelona: Editorial Icaria.

Unidas, N. (s. f.). Teoría y práctica de la Seguridad Humana. Recuperado el 15 de julio de 2016, de https://docs.unocha.org/sites/dms/HSU/Publications $\% 20$ and $\% 20$ Products/Human \%20Security \%20Tools/Human \% 20 Security\%20in\%20Theory\%20and\%20Practice\%20Spanish.pdf

United States (2002). National Security Strategy. Washington D.C .

Wallerstein, I. (2001). Después del Liberalismo. México D. F.: Siglo XXI.

Waltz, K. (1979). Teoría de la politica internacional. New York: McGraw Hill.

Zizek, S. (2002). Bienvenidos al desierto de lo real. Madrid: Ediciones Akal.

\section{(Footnotes)}

1 Documento completo: http://mepoforum.sk/wp-content/uploads/2015/08/ NDS-RF-2009-en.pdf

2 Documento en la página web del Kremlin. http://en.kremlin.ru/events/ president/news/51129 
